\title{
FishMap: A Community Resource for Zebrafish Genomics
}

\author{
Rajeshwari Meli, Abhiranjan Prasad, Ashok Patowary, Mukesh K. Lalwani, Jayant Maini, \\ Meenakshi Sharma, Angom Ramcharan Singh, Gaurav Kumar, Vaibhav Jadhav, \\ Vinod Scaria, and Sridhar Sivasubbu
}

\begin{abstract}
An enormous amount of information on a genomics scale is available for zebrafish (Danio rerio), which is a well-studied model organism for human diseases. However, a majority of this annotation is scattered in obscure data sources. There have been limited efforts to present it on a unified and integrated platform, which would help to understand the biological processes in this organism better. FishMap is a unified and centralized resource for storage, retrieval, and display of genomic information of zebrafish. The datasets have been methodically collected from various resources and supplementary information of publications and mapped to the zebrafish genome. The data are organized into nine major sections, which include comparative genomics, mapping and sequencing, gene and gene predictions, expression and regulation, and variation and repeats. A number of unique sections have been incorporated, which include tracks on noncoding gene annotation, location of retrovirus/transposon integrations in the genome, and their flanking genomic sequences and novel transcripts. The datasets are linked to related data sources. FishMap is built on the Gbrowse, which is a part of the Generic Model Organism Database Consortium Project. The resource also features a Web-based BLAST server for sequence homology search and a gene ID converter that would enable users to sift through different interchangeable gene annotation identifier systems. The database is amenable to programmatic access through the Distributed Annotation System as well as BioMoby protocols, thus making it a central community resource that can be integrated with existing data mining and analysis workflows. We hope that FishMap would be an integral resource for community participation in zebrafish genomics. The resource is freely available at http://miracle.igib.res.in/fishmap, or at http://fishmap.igib.res.in.
\end{abstract}

\section{Introduction}

$\mathbf{Z}$ EBRAFISH (DANIO RERIO) is a wellestablished model organism for investigating vertebrate development, organ function, and diseases. Characteristics that have made zebrafish a popular model system include small size at maturation, relatively short generation time, ex utero development, and optical transparency of its embryos. Zebrafish also shows a high degree of genetic and anatomical conservation with humans. Large tracks of zebrafish chromosomes are in synteny with human genome. ${ }^{1}$ Further, sequencing of the zebrafish genome coupled with rapid advances in tools for manipulating the genome $\mathrm{e}^{2-9}$ has enabled zebrafish to become a preeminent model system for investigating human disease and pathophysiological processes.

An enormous amount of information on a genomics scale is available for zebrafish. Whole genome sequence of zebrafish is publicly available at Ensembl (http://www.ensembl.org/ Danio_rerio/index.html). Ensembl also provides 
information on gene and gene predictions, manual annotation, comparative genomics, SNPs, and has a provision for conducting sequence homology searches against all zebrafish expressed sequence tags (ESTs) and cDNA among several other features. Other resources featuring genomic-level information for zebrafish include the University of California Santa Cruz (UCSC) genome browser (http://genome.ucsc.edu) and NCBI Map Viewer (http://www.ncbi.nlm.nih .gov/mapview/map_search.cgi?taxid $=7955$ ), both of which house extensive genome-level information. A vast collection of expressed sequence tags and cDNAs from numerous developmental stages and tissue types offers insights into the expression pattern and cellular roles and functions in addition to providing comprehensive sets of high-quality cDNA libraries. Several commercial and academic sources have generated DNA microarray data for expression profiling in zebrafish. Apart from these, there exists a large collection of zebrafish mutants (both chemical and insertional mutants), gene and enhancer trap lines, morpholinos, meiotic and radiation hybrid maps, and several ortholog predictions for zebrafish. In addition, an excellent zebrafish model organism database (Zebrafish Information Network [ZFIN]) provides genetic and developmental information and houses a large amount of gene-centered data on zebrafish. ${ }^{10}$ Ensembl in collaboration with ZFIN also has high-quality annotation for a limited set of genes through VEGA. The combined efforts of all these resources will produce a reference set of genes with high-quality annotations (at a macro level) for the zebrafish research community. However, it is now evident that many genes will have multiple splice forms with diverse expression levels in different tissue types, each having its own unique biological significance. In such cases, one would need a platform for "micro/ fine annotation" of genes and for linking them to biology. We envisage FishMap to bridge the fine annotation gap that currently exists between gene-centered resources, such as ZFIN, and genome-centered resources, such as Ensembl and UCSC databases. FishMap will be an individual investigator-driven annotation resource where the fine-tuning of gene annotation will be done by individual investigators on prior information generated by ZFIN, Ensembl, or
UCSC databases. FishMap intends to serve as a complementary resource to the ZFIN and other genome browsers.

Further, a majority of this genetic and genomic annotation is generated by individual laboratories, and there have been no large-scale efforts to standardize data exchange formats or collect them in a systematic way. This has resulted in a situation where a large amount of genome-scale information on zebrafish is available in obscure sources and nonstandard formats, which are not easily amenable for large-scale and integrative analysis. Availability of these genome-scale datasets on a unified and integrated platform would help tremendously in understanding biological processes in a holistic way. As the genome-level data from largescale genetic, genomic, and chemical screens become available, one would need an integrated database for displaying genomic, expression, and protein data, and for linking genotypic and phenotypic information to make meaning out of these datasets. In this regard, a centralized, Web-accessible, integrated database would provide a valuable resource for common access to these data while allowing sophisticated data-mining queries. With these ideas in mind, we have developed FishMap, a platform that would allow data integration and visualization for better interpretation of zebrafish-centered biological processes.

FishMap is an attempt to integrate genomic information of zebrafish in a systematic way. FishMap is built on the Gbrowse, which is a part of the Generic Model Organism Database. This site provides a user-friendly access to several zebrafish genomic resources that were previously not amenable for display in a genomic context, along with a number of unique sections on noncoding gene annotation, genomic integrations, and novel transcripts. FishMap is a community resource and would accept submission of datasets from the community for integration. The resource can be availed online at http://miracle.igib.res.in/fishmap, or at http://fishmap.igib.res.in.

\section{Data Sets and Data Curation}

The zebrafish genome sequence (version Zv7), generated by the Danio rerio sequencing 
project at Sanger Institute, was used for all annotations. The latest genome sequence was downloaded from the UCSC genome browser. The datasets were methodically collected from various publicly available Websites, resources, and supplementary information of publications and were mapped to the zebrafish genome (see Supplemental Table 1 [www.liebertpub.com/ zeb] for details regarding each data track). Sequence datasets were mapped to the genome using BLAT. ${ }^{11}$ Datasets with position mapping to previous builds of the genome were mapped to the present build using the Liftover tool and mappings available at UCSC genome browser. In version 1.0 of the FishMap, data are organized into nine major sections, which include mapping and sequencing, gene and gene predictions, expression and regulation, comparative genomics, and variation and repeats. Additional data sets were created from the genomics sequence data via a series of automated analyses, including noncoding gene annotation, location of retrovirus and transposon integrations, and novel transcripts. Currently, there are about 50 data tracks and analysis options available on the FishMap (Table 1).

Data tracks for detection of human homolog in the zebrafish genome for annotated genes have been incorporated in the current version.
This feature offers a single-window operation for identifying human homolog in zebrafish while conducting genomic searches. Data tracks for genes mutated in the chemical mutagenesis screens using ethylnitrosourea (ENU) have been displayed along with tracks representing retroviral and transposon-mediated insertions from various laboratories. Currently, several groups are undertaking genome-wide gene trapping and mutagenesis projects in zebrafish. FishMap will allow the collection, display, and query of a large volume of genetic and phenotypic information obtained from such projects. In addition, FishMap also displays over 400 novel experimentally validated transcripts generated by gene trapping in zebrafish (A. Patowary and S. Sivasubbu, unpublished data). These will be used for identifying new transcripts and for annotation of the existing genes in the zebrafish genome.

\section{Genome Browser}

FishMap genome browser is based on the generic genome browser (Gbrowse), which is part of the GMOD consortium. ${ }^{12}$ Gbrowse is a powerful Web-based application for display of genome-scale datasets for any organism. The database is scalable and has a variety of plugins

Table 1. Summary of the Data Sets Incorporated in FishMap Genome Browser

\begin{tabular}{ll}
\hline Category & \multicolumn{1}{c}{ Features available } \\
\hline Gene and gene prediction & $\begin{array}{l}\text { Ensembl gene, RefSeq gene, VEGA gene, Ensembl gene structure, MGC gene } \\
\text { structure, CONTRAST gene predictions, GEN ID v1.3, Reference gene structure, } \\
\text { ZGC gene, other mRNAs }\end{array}$ \\
Comparative genomics & $\begin{array}{l}\text { Fugu chain, Human net, Mouse chain, Tetraodon net, Fugu net, Medaka chain, } \\
\text { Mouse net, Human chain, Medaka net, Tetraodon chain }\end{array}$ \\
Mapping and sequencing & Assembly, Contigs, Gap \\
Gene expression & EST-UCSC, Transcripts with Affymetrix probe sets, Transcripts with wild-type \\
array probe sets, ZFMODELS Carp EST & ZNOMICS Retrovirus insertions, Sridhar lab transposon insertions, Genes mutated \\
Insertions and phenotypes & by ENU, Morpholino, ZF MODELS Tilling data \\
Variations and repeats & dbSNP, Interrupted Repeats, RepeatMasker, Simple Repeats \\
Noncoding RNA & ANCORA Highly Conserved Noncoding Elements (HCNEs), miRBase microRNA \\
& targets, tRNAscan SE predictions, ECRbase evolutionary conserved regions, \\
miRBase v10 microRNAs & NXsensor, Quadfinder G-Quadruplex Motifs \\
Regulatory regions & Human ortholog genes in zebrafish-predicted by Homologene; Human ortholog \\
Zebrafish-human orthologs & genes in zebrafish-predicted by Inparanoid \\
Overview and analysis & Chromosome overview, DNA/GC content, CMapDumper, BatchDumper, \\
(including plugins) & FastaDumper, RestrictionAnnotator, FilterTest, GFFDumper
\end{tabular}




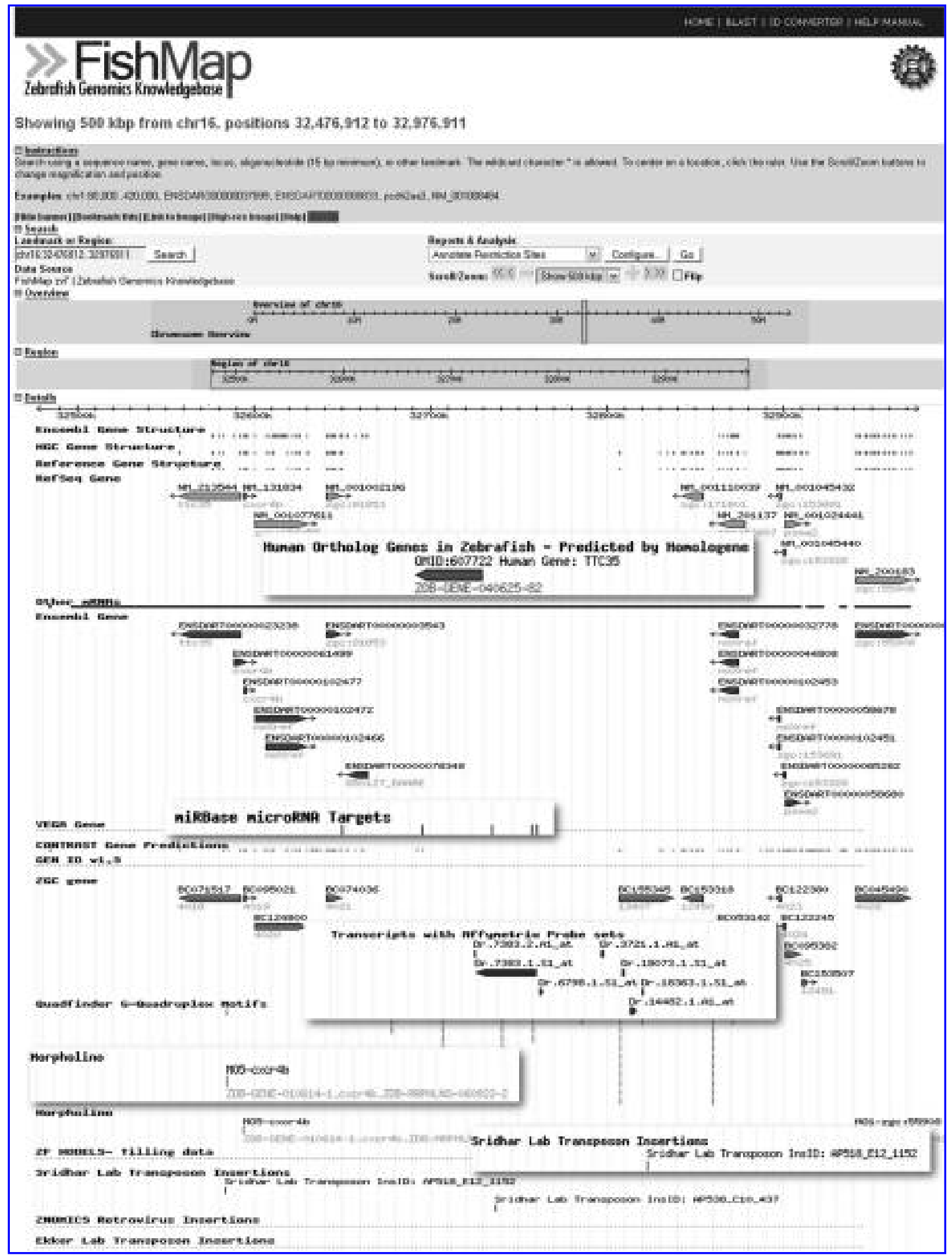

FIG. 1. A screenshot of the FishMap genome browser depicting a portion of chromosome 16 with various data sets that have been mapped to the zebrafish genome. Data tracks representing transcripts with Affymetrix probe sets, miRNA target, Morpholino, and Transposon insertions have been highlighted. 
for in-depth analysis and data export. Genomic positions can be keyed in or selected from the overview panel, and tracks can be selected from the list of available tracks for visualization. Each data point is linked to relevant details or Web pages. FishMap also allows users to upload their annotations for viewing and analysis based on the existing tracks. The plugins on Gbrowse enable users to map restriction enzyme cutting sites of their choice as well as download relevant parts of the sequence along with the annotations for further analysis in GFF or FASTA format. Users can also permanently create links to predefined views for chromosomal loci and sets of tracks, and also export high-resolution images. Figure 1 describes a screenshot of the FishMap genome browser depicting a portion of chromosome 16 with various data sets that have been mapped to the zebrafish genome.

\section{Tools and Analysis Options Integrated}

To enhance the usability of the genome browser, a number of tools have been incorporated in FishMap. The resource hosts a public BLAST $^{13}$ server for sequence homology searches. This would enable users to find genomic features and information pertaining to regions in the zebrafish genome mapping to their sequences of interest. A large number of interchangeable identifiers are commonly used for zebrafish genes and transcripts, and sifting through the various identifiers is a daunting task. FishMap integrates an ID conversion tool that would simplify the process. Almost all commonly used identifiers can be converted to another using the batch query option. FishMap also features plugins, which allow users to download datasets in GFF format, which is compliant with most other genome browsers. The resource accepts datasets and suggestions/submissions, and features a Web-blog for announcements.

\section{Future Directions}

The fundamental aim of FishMap is to develop a unified resource for zebrafish genomic information. This would be a starting point for further community participation in fine annotation of the zebrafish genome. Such a resource is best utilized when it is open to active participation by all members of the community. We encourage submission and suggestion of datasets from the community. We have also envisaged updating the FishMap database on a continuous basis. In the immediate future, we will incorporate a wealth of information on retroviral insertions and noncoding RNAs, apart from incorporating genome-scale predictions from various labs.

Further, we will be integrating tools to enable large-scale data mining by incorporating a seamless search and retrieval system that would support complex queries. We would also invest in open standards and schemas for data annotation and exchange that would enable seamless access of datasets housed in FishMap by other data browsers, databases, and analysis tools, thereby serving as a complementary resource to existing data sources. We have initiated the process by integrating a Distributed Annotation System server with the resource.

We hope FishMap would act as a catalyst for activities such as fine annotation of the zebrafish genome, linking of genes to the biology by the experts who work on them and provide a way to link genotype to phenotype by members of the community who generated the data in the laboratory.

\section{Acknowledgments}

The research was supported by a grant from Council of Scientific and Industrial Research, India (Grant No. FAC002). We are particularly grateful to lab members for their valuable inputs and encouragement. We thank members of the zebrafish community for freely sharing their data.

\section{Conflict of Interest Statement}

None declared.

\section{References}

1. Barbazuk WB, Korf I, Kadavi C, Heyen J, Tate S, Wun E, et al. The syntenic relationship of the zebrafish and human genomes. Genome Res 2000;10:1351-1358.

2. Haffter P, Granato M, Brand M, Mullins MC, Hammerschmidt M, Kane DA, et al. The identification of genes with unique and essential functions in the development of the zebrafish, Danio rerio. Development 1996;123:1-36. 
3. Amsterdam A, Burgess S, Golling G, Chen W, Sun Z, Townsend $\mathrm{K}$, et al. A large-scale insertional mutagenesis screen in zebrafish. Genes Dev 1999;13:2713-2724.

4. Nasevicius A, Ekker SC. Effective targeted gene "knockdown" in zebrafish. Nat Genet 2000;26:216220.

5. Balciunas D, Davidson AE, Sivasubbu S, Hermanson SB, Welle Z, Ekker SC. Enhancer trapping in zebrafish using the Sleeping Beauty transposon. BMC Genomics 2004;5:62.

6. Kawakami K, Takeda H, Kawakami N, Kobayashi M, Matsuda N, Mishina MA. Transposon-mediated gene trap approach identifies developmentally regulated genes in zebrafish. Dev Cell 2004;7:133-144.

7. Parinov S, Kondrichin I, Korzh V, Emelyanov A. Tol2 transposon-mediated enhancer trap to identify developmentally regulated zebrafish genes in vivo. Dev Dyn 2004;231:449-459.

8. Sivasubbu S, Balciunas D, Davidson AE, Pickart MA, Hermanson SB, Wangensteen KJ, et al. Gene-breaking transposon mutagenesis reveals an essential role for histone H2afza in zebrafish larval development. Mech Dev 2006;123:513-529.

9. Wienholds E, Eeden FV, Kosters M, Mudde J, Plasterk RHA, Cuppen E. Efficient target-selected mutagenesis in zebrafish. Genome Res 2003;13:2700-2707.

10. Sprague J, Bayraktaroglu L, Clements D, Conlin T, Fashena D, Frazer $\mathrm{K}$, et al. The zebrafish information network: the zebrafish model organism database. Nucleic Acids Res 2006;34:D581-D585.
11. Kent WJ. BLAT-the BLAST-like alignment tool. Genome Res 2002;12:656-664.

12. Stein LD, Mungall C, Shu S, Caudy M, Mangone M, Day A, et al. The generic genome browser: a building block for a model organism system database. Genome Res 2002;12:1599-1610.

13. Altschul SF, Gish W, Miller W, Myers EW, Lipman DJ. Basic local alignment search tool. J Mol Biol 1990; 215:403-410.

Address reprint requests to: Vinod Scaria, MBBS

Institute of Genomics and Integrative Biology Council of Scientific and Industrial Research Mall Road Delhi 11007 India

E-mail: vinods@igib.in

Sridhar Sivasubbu, Ph.D. Institute of Genomics and Integrative Biology Council of Scientific and Industrial Research

Mall Road

Delhi 11007

India

E-mail: sridhar@igib.in 